\title{
BCAT1 Promotes Lung Adenocarcinoma Progression Through Enhanced Mitochondrial Respiration and NF-KB Pathway Activation
}

\section{Mengdan Yu}

Henan Institute of Medical and Pharmaceutical Sciences, Zhengzhou University, Zhengzhou, China

Qianwei Zhao

Henan Institute of Medical and Pharmaceutical Sciences, Zhengzhou University, Zhengzhou, China Jinxia Li

School of Basic Medical Sciences, Academy of Medical Sciences, Zhengzhou University, Zhengzhou, China

\section{Fang Xu}

Henan Institute of Medical and Pharmaceutical Sciences, Zhengzhou University, Zhengzhou, China

\section{Zhibiao Zhang}

School of Life Sciences, Zhengzhou University, Zhengzhou, China

\section{Yixian Liu}

Henan Institute of Medical and Pharmaceutical Sciences, Zhengzhou University, Zhengzhou, China

\section{Liping Dai}

Henan Institute of Medical and Pharmaceutical Sciences, Zhengzhou University, Zhengzhou, China

\section{Bingxia Zhang}

School of Life Sciences, Zhengzhou University, Zhengzhou, China

Jianying Zhang

Henan Institute of Medical and Pharmaceutical Sciences, Zhengzhou University, Zhengzhou, China Jintao Zhang ( $\square$ zhangjt_66@126.com )

Henan Institute of Medical and Pharmaceutical Sciences, Zhengzhou University, Zhengzhou, China

\section{Research Article}

Keywords: BCAT1, LUAD, BCAAs, mitochondrial respiration, NF-kB pathway

Posted Date: June 18th, 2021

DOI: https://doi.org/10.21203/rs.3.rs-604725/v1

License: (c) (1) This work is licensed under a Creative Commons Attribution 4.0 International License. Read Full License 


\section{Abstract}

BCAT1 is up-regulated and acts as an oncogenic factor in many types of cancers, but its role in lung adenocarcinoma (LUAD) development is not clearly understood. Here we found BCAT1 protein level was up-regulated in tumor tissues, which was positively associated with TNM stage and local lymph node metastasis of LUAD patients. BCAT1 knockdown inhibited cell growth and mobility while BCAT1 overexpression promoted LUAD development both in vitro and in vivo. BCAAs metabolism and mitochondrial respiration were enhanced in BCAT1 overexpression cells, which were more sensitive to Leucine and Isoleucine supplements, compared to control cells. Moreover, RNA sequencing analysis suggested that differentially expressed genes (DEGs) in BCAT1 overexpression LUAD cells were enriched in metabolism, signal transduction, and immune response processes, and BCAT1 overexpression decreased NFKBIB mRNA level that induced NF- $\kappa$ B pathway activation in LUAD cells. As an inhibitor of NF- $K B$ pathway, ammonium pyrrolidinedithiocarbamate (PDTC) treatment predominately counteracted the effect of NF- $K B$ pathway activation and inhibited LUAD cells proliferation and migration, especially cells with BCAT1 overexpression. Taken together, our findings point a key role for BCAT1 in promoting LUAD development through metabolic reprogramming and NF- $K B$ pathway activation, which provides promising molecular biomarker and therapeutic targets for LUAD diagnosis and treatment.

\section{Introduction}

Lung cancer is one of the most common and malignant cancers in the world, and non-small cell lung cancer (NSCLC) takes around $85 \%$ of all the cases ${ }^{1}$. NSCLC mainly includes LUAD and squamous cell carcinoma (SCC) ${ }^{2}$. Among NSCLC cases, LUAD accounts for about $60 \%$ and remains a major cause of cancer-related mortality worldwide ${ }^{3,4}$. In recent years, increasing researches on the pathobiology and progression of lung cancer have been performed and many genes have been identified as potential drivers and targets for therapy ${ }^{5-9}$. However, the cure and prognosis of lung cancer patients are hardly improved $^{10,11}$. Therefore, it is necessary to reveal the precise mechanism of lung cancer development and develop more efficient diagnostic and therapeutic methods for clinical lung cancer patients.

Among the Branched-chain aminotransferases (BCAT) enzymes, BCAT1 is an enzyme that plays an important role in branched-chain amino acids (BCAAs) metabolism process. BCAAs including leucine, isoleucine and valine are essential nutrients for protein synthesis and cell growth, which can be consumed to provide energy for cancer cells growth and proliferation ${ }^{12,13}$. Increasing evidence showed that BCAT1 is a vital and well researched enzyme which is closely associated with cancers development. It was found that BCAT1 was up-regulated in many kinds of cancers, which has been proposed as a prognostic cancer cell marker ${ }^{14-16}$. Besides, researches showed that BCAT1 up-regulation promoted cancer cell proliferation, cell cycle progression, differentiation, apoptosis, invasion and metastasis in numerous types of cancers, such as gastric cancer, colorectal cancer, prostate cancer, breast cancer, ovarian cancer, hepatocellular cancer, and so on ${ }^{17-20}$. As an important enzyme, BCAT1 and its metabolite can interact with metabolism process in cancer cells through different ways, such as mitochondrial 
biogenesis, fatty acid metabolism and so on. For example, glutamate generated from BCAAs metabolism is an important source of $a$-ketoglutarate, which is an intermediate product of TCA cycle and provides energy for cell growth and proliferation ${ }^{21}$. It is also found that BCAT1 promotes the growth of breast cancer cells through improving mTOR-mediated mitochondrial biogenesis and function, while BCAT1 suppression results in downregulation of numerous genes implicated in lipid production and protein synthesis, and finally inhibits tumor growth ${ }^{12,16}$. Taken together, BCAT1 plays an important role in cancer development, and making it clear of its functional mechanism during LUAD development possibly promotes the clinical treatment of LUAD patients.

In this study, we found BCAT1 was overexpressed in LUAD cancer tissues, compared to the normal lung tissues of LUAD patients. The in vitro and in vivo studies showed that BCAT1 overexpression promoted LUAD cells proliferation, migration and invasion. Besides, we found that BCAT1 overexpression promoted BCAAs metabolism and mitochondrial respiration of LUAD cells, and transcriptomic study indicated that NF- $K B$ signaling pathway was activated in BCAT1 overexpression LUAD cells. Moreover, treatment of LUAD cells with PDTC, which is an inhibitor of NF- $K B$ pathway, prominently inhibited cell proliferation and invasion abilities. In conclusion, our findings offer insights into the role of BCAT1 in NF- $K$ B pathway activation during LUAD development, which might provide new methods for clinical diagnose and treatment of LUAD patients.

\section{Results}

\section{BCAT1 was up-regulated in tumor tissues of LUAD patients}

To evaluate the significance of BCAT1 during LUAD development, we investigated the expression levels of BCAT1 in tumor and para-carcinoma normal lung tissues from clinical LUAD patients. TMAs containing 86 pairs of tissue specimens were examined by IHC staining using human BCAT1 antibody. The positive rates were scored as $0,1-2$, and 3-4 for negative, weak positive, and positive staining, respectively. As shown in Fig. 1A, the frequency of positive staining of BCAT1 in tumor tissues was significantly higher (69 of $86[80 \%])$ than that in normal tissues (14 of 86 [16\%]), while there existed $5(0.5 \%)$ and 61 cases $(71 \%)$ in tumor and normal tissues where BCAT1 were negative staining, respectively. According to the "staining intensity score" and the "staining positive rate score" from IHC results, the expression level of BCAT1 was significantly higher in LUAD tumor tissues compared to normal lung tissues (Fig. 1B, $p<$ 0.0001). Furthermore, statistical analysis of LUAD specimens indicated that BCAT1 expression level was positively associated with local lymphatic metastasis $(p=0.0304)$ and TNM stage $(p=0.0189)$, but not with the gender, age, tumor size, distant metastasis, and overall survival (Fig. 1C). Taken together, these results indicated that BCAT1 could be a promising biomarker for clinical LUAD diagnosis, and its upregulation possibly contributed to LUAD initiation and progression. 


\section{BCAT1 knockdown inhibited proliferation, migration, and invasion of LUAD cells in vitro}

Next, we detected the expression levels of BCAT1 in immortalized normal lung cell line (BEAS-2B) and LUAD cell lines including NCI-H1975, H1299, A549 and HCC827 by western blot, and results showed that BEAS-2B and H1299 showed high expression level of BCAT1 while NCl-H1975 and HCC827 showed low expression level of BCAT1. Besides, A549 performed moderate expression level of BCAT1, which was chosen for functional study of BCAT1 in LUAD development (Figure S1). Then lentiviral shRNA vector (BCAT1-sh) targeting BCAT1 were constructed and transfected into A549 cells. Western blot and qRT-PCR analysis in Fig. 2A showed that expression level of BCAT1 were knocked down by more than $60 \%$ with BCAT1-sh, compared to the cells transfected with empty vector (sh-con). Then cell proliferation abilities of BCAT1 knockdown and sh-con LUAD cells were detected by xCELLigence Real-Time Cell Analyzer (RTCA)MP system. As shown in Fig. 2B, BCAT1 knockdown significantly decreased proliferation rate of LUAD cells in vitro. Besides, the colony formation assay in Fig. $2 \mathrm{C}$ showed that colony numbers were decreased in BCAT1 knockdown LUAD cells. Furthermore, wound healing, migration and matrigel invasion assays were performed in BCAT1 knockdown and control LUAD cells, and results in Fig. 2D-F showed that the wound closure ability was decreased and the migratory and invasive cell numbers were markedly reduced, which was consistent with the results that E-cadherin was up-regulated while Vimentin was down-regulated in BCAT1 knockdown cells. Collectively, these results demonstrated that BCAT1 suppression could inhibit tumorigenesis of LUAD cells in vitro.

\section{BCAT1 overexpression promoted tumorigenesis of LUAD cells both in vitro and in vivo}

Then we constructed the A549 cell line that stably overexpressing BCAT1 with lentivirus method. The expression levels of exogenous BCAT1 were demonstrated by western blot and qRT-PCR analysis in Fig. 3A. Then these two groups of cells were subjected to cell growth and colony formation assays (Fig. 3B and 3 C), and results showed that the cell proliferation rate and colony formation ability of BCAT1transfected cells were both much higher than those of control cells (Lv-con). Besides, the function of BCAT1 on motility and invasiveness of LUAD cells were investigated. The wound healing assay showed that overexpression of BCAT1 enhanced LUAD cells migration at the edge of scratched regions and significantly accelerated the speed of wound closure (Fig. 3D). Migration and matrigel invasion assays showed that the number of BCAT1-transfected cells migrating through the filter membrane remarkably increased compared to that of Lv-con cells (Fig. 3E). Besides, it was found that the expression level of Ecadherin was decreased while Vimentin was increased in BCAT1 overexpression cells (Fig. 3F). These results suggested that overexpression of BCAT1 enhanced the mobility and invasion capability of LUAD cells in vitro. Furthermore, to study the influence of BCAT1 on LUAD development in vivo, we established the xenograft mice model and A549 cells with BCAT1 overexpression and control cells were implanted subcutaneously into nude mice, respectively. Five weeks later, mice were sacrificed and the tumors were 
collected for analysis. Results in Fig. 4A-C showed that the volumes and weights of LUAD tumors from BCAT1 overexpression group were larger and higher than those of control group. Additionally, IHC analysis was performed with the LUAD tumors and it showed that BCAT1 overexpression was maintained in the xenografts (Fig. 4D). In conclusion, these results suggested that BCAT1 overexpression promoted LUAD tumor formation and development both in vitro and in vivo.

\section{BCAT1 overexpression enhanced BCAAs consumption and mitochondrial respiration of LUAD cells}

Since BCAT1 is an important enzyme during BCAAs (Leucine, Isoleucine, Valine) metabolism process, we examined the effects of BCAAs supplementation on BCAT1 overexpression LUAD cells (Fig. 5A). It showed that the cell proliferation ability was enhanced with increasing BCAAs concentration. Moreover, we examined the sensitivity of BCAT1 overexpression and control cells to BCAAs supplementation, respectively. Results showed that compared to control cells, the cell proliferation abilities of BCAT1 overexpression cells were higher with Leucine and Isoleucine supplementation, while there was no difference in cell proliferation abilities between BCAT1 overexpression and control cells with Valine supplementation (Fig. 5B). BCAAs metabolism mainly provided metabolic intermediates for TCA cycle and mitochondrial oxidative phosphorylation that contributed to energy generation or biosynthesis (Fig. 5C), then we detected the mRNA expression level of several important genes associated with BCAAs metabolism in BCAT1 overexpression and control LUAD cells. Results in Fig. 5D showed that the transcription levels of genes promoting BCAAs metabolism including BCKDHA, BCKDHB, and ECHS1 increased and BCAAs metabolism inhibitor BCKDK was down-regulated in BCAT1 overexpression cells. It indicated that BCAAs metabolism possibly contributed to energy production in BCAT1 overexpression cells. Then we measured the effect of BCAT1 overexpression on oxygen consumption of LUAD cells. The mitochondrial respiration was assessed by measurement of OCR, which was increased in BCAT1 overexpression cells while BCAT1 inhibition decreased OCR (Fig. 5E and F). Taken together, these results proved that BCAT1 overexpression enhanced BCAAs metabolism and mitochondrial respiration to promote LUAD development.

\section{RNA-seq and bioinformatics analysis of DEGs in BCAT1 overexpressed LUAD cells}

To further study the mechanism of BCAT1 in promoting LUAD development, RNA-seq was performed in BCAT1 overexpressed and control LUAD cells, respectively. The global transcriptome maps showed that a total number of 17503 genes were detected both in BCAT1 overexpressed and control LUAD cells. About 440 DEGs were identified, among which 139 genes were down-regulated and 301 genes were upregulated in BCAT1 overexpressed LUAD cells (Fig. 6A). Then GO analysis was performed with these DEGs based on biological process, cellular component, and molecular function (Fig. 6B). Biological process analysis indicated that DEGs were enriched in cellular process, biological regulation, response to 
stimulus, multicellular organismal process, signaling, developmental process, metabolic process, and localization. Cellular component analysis showed that DEGs were mainly located in cell, organelle, membrane, and membrane part. Besides, molecular function analysis showed that DEGs were mostly characterized by functions including binding and catalytic activity. To better understand the cellular processes regulated by BCAT1 overexpression in LUAD cells, KEGG pathway classification analysis was carried out, and results in Fig. $6 \mathrm{C}$ showed that DEGs were primarily clustered in six categories, among which DEGs were mostly associated with signal transduction, cancer development, metabolism and immune system. Consistently, we performed KEGG functional enrichment analysis in Fig. 6D, and it indicated that DEGs were mainly enriched in metabolic processes, AGE-RAGE signaling pathway, cell adhesion and migration, and so on.

\section{NF-KB signaling pathway was activated in BCAT1 overexpression LUAD cells}

Among the DEGs, RNA-seq results showed that NFKBIB was significantly downregulated in BCAT1 overexpressed LUAD cells. Since NFKBIB combines with NF- $K B$ in the cytoplasm and prevents its transportation into nucleus, inhibiting NF- $K B$ signaling pathway activation. Downregulation of NFKBIB possibly indicated activation of NF- $K B$ signaling pathway in BCAT1 overexpressed LUAD cells. To verify this hypothesis, we first detected the transcription level of NFKBIB by qRT-PCR, and found that it was decreased in BCAT1 overexpressed cells while increased in BCAT1 knockdown cells, as compared with control cells (Fig. 7A). Then the expression levels of proteins involved in NF- $K B$ signaling pathway were investigated, as shown in Fig. 7B, BCAT1 overexpression resulted in higher expression levels of IKK $a / \beta$, phosphorylated p65 (p-p65) and lower expression level of IKB $a$. These findings suggested that BCAT1 overexpression promoted NF- $\kappa$ B signaling pathway activation in LUAD cells.

\section{PDTC treatment inhibited cell proliferation, migration and invasion of BCAT1 overexpression LUAD cells in vitro}

To verify the finding that BCAT1 overexpression activated NF- $K B$ pathway to promote LUAD development, then we treated BCAT1 overexpression and control LUAD cells with PDTC, which is an inhibitor of NF- $K B$ pathway activation. Results in Fig. 7C showed that PDTC treatment inhibited the proliferation ability of both BCAT1 overexpression and control LUAD cells, and the inhibiting effect was positively related to the concentration of PDTC. Besides, compared with control LUAD cells, BCAT1 overexpression LUAD cells were much more sensitive to PDTC treatment, which further indicated activation of NF- $K B$ pathway in BCAT1 overexpression LUAD cells. Moreover, we detected the effect of PDTC on migration and invasion abilities of BCAT1 overexpressed LUAD cells. Results in Fig. 7D showed that PDTC treatment significantly decreased the number of migratory and invasive cells. In conclusion, these results suggested that BCAT1 overexpression could promoted LUAD development through NF- $K$ B pathway activation, which could be 
counteracted by the treatment of NF- $K B$ pathway inhibitor. This finding provided promising therapeutic method for clinical LUAD cure.

\section{Discussion}

To study the relationship between BCAT1 and LUAD development, here we firstly detected the expression level of BCAT1 in tumor and normal lung tissues from LUAD patients. It showed that BCAT1 was significantly overexpressed in tumor tissues, and its expression level was associated with TNM stage and local lymphatic metastasis of clinical LUAD patients, but not with distant metastasis and overall survival. These findings were consistent with an earlier research that BCAT1 was up-regulated in tumor tissues of lung cancer patients and BCAT1 overexpression promoted NSCLC development ${ }^{22}$. Besides, here we found BCAT1 was overexpressed in several LUAD cell lines. These findings suggested that BCAT1 could be taken as a promising biomarker for lung cancer diagnosis including LUAD, and further indicated BCAT1 as a carcinogenic factor during LUAD development.

The function and mechanism study of BCAT1 in LUAD development showed that BCAT1 overexpression promoted tumorigenesis of LUAD cells both in vitro and in vivo. BCAT1 is an enzyme catalyzing the metabolism of BCAAs. It has been proved that BCAT1 overexpression promoted cancers development through regulating metabolic progresses, such as oxidative phosphorylation, fatty acid and amino acid metabolism ${ }^{23-25}$. Consistently, here we found that BCAT1 overexpression enhanced BCAAs metabolism and mitochondrial respiration in LUAD cells. Moreover, transcriptomic analysis of BCAT1 overexpression and LV-con A549 cells showed that the DEGs induced by BCAT1 overexpression were mainly clustered into two main categories including metabolism and signal transduction. Among the metabolism associated DEGs, genes were primarily involved in fatty acid synthesis and differentiation. These results suggested that BCAT1 overexpression enhanced BCAAs metabolism, and lead to enhancement of mitochondrial respiration and fatty acid synthesis to promote LUAD cells growth. Besides metabolic reprogramming, KEGG pathway enrichment showed that DEGs were also enriched in AGE-RAGE signaling pathway. AGEs were a kind of metabolites produced from proteins and lipids that induced signaling transduction through RAGE receptors in several kinds of diseases including cancer, metabolic diseases, and so on ${ }^{26,27}$. BCAT1 and enhanced BCAAs consumption were reported to be involved in several signaling pathways associated with cancer development, such as MYC, mTOR, $\beta$-cactenin, NF- $\kappa \mathrm{B}$ and so on ${ }^{15,16,28}$. Taken together, these results indicated that BCAT1 overexpression promoted metabolism reprogramming of LUAD cells, and lead to signal transduction processes to promote LUAD development.

NF- $K B$ is a nuclear transcription factor which regulates expression of a large number of genes that are critical for the regulation of apoptosis, tumorigenesis, inflammation, and metabolic processes ${ }^{29}$. Increasing researches showed that NF- $\kappa$ B pathway activation was found in the tumor tissues of many types of cancers including lung cancer ${ }^{30,31}$. Besides, it was found that NF- $K B$ pathway activation promoted cancer development through enhancing cell proliferation, invasion, and angiogenesis, inhibiting cell apoptosis, inducing metabolism reprogramming, and regulating inflammation and immune response, 
and so on ${ }^{32-34}$. Therefore, more and more inhibitors of NF- $K B$ pathway were being exploited and NF- $\kappa B$ pathway inhibition was taken as a promising method for cancer treatment ${ }^{35-38}$. In this study, we found that NF- $\kappa$ B pathway was activated in BCAT1 overexpression LUAD cells, and NF- $K B$ pathway inhibitor of PDTC treatment could significantly decreased proliferation and invasion abilities of LUAD cells while BCAT1 overexpression LUAD cells seemed to be more sensitive to PDTC treatment. These findings indicated that NF- $K B$ pathway activation was a pivotal reason to promote LUAD progression induced by BCAT1 overexpression, and proved that NF- $K$ B pathway inhibition could prevent LUAD development in vitro. Furthermore, it provided possibility for application of NF- $k B$ pathway inhibitors for precise treatment of LUAD patients with BCAT1 overexpression in tumor tissues.

In conclusion, this study revealed that BCAT1 was up-regulated in tumor tissues of LUAD patients. BCAT1 overexpression promoted LUAD development both in vitro and in vivo through enhancing BCAAs consumption and mitochondrial respiration, and finally activated NF- $\kappa$ B pathway, which could be counteracted by PDTC treatment (Fig. 8). These findings suggested BCAT1 as a promising biomarker for LUAD diagnosis, and provided NF- $K B$ pathway inhibitors as potential therapeutic methods for LUAD treatment.

\section{Material And Methods}

\section{Cell culture}

The immortalized normal lung cell line (BEAS-2B) and LUAD cell lines including NCl-H1975, H1299, A549 and HCC 827 were cultured in RPMI-1640 or DMEM with $10 \%$ FBS and incubated at $37^{\circ} \mathrm{C}$ in a humidified incubator with $5 \% \mathrm{CO}_{2}$. BEAS-2B was purchased from the Chinese Academy of Sciences Typical Culture Preservation Committee Cell Bank. A549 was purchased from Genetic Testing Biotechnology Corporation. HCC827 was gifted by Proteomics and Signal Transduction Center, Zhengzhou University. H1299 and $\mathrm{NCl}-\mathrm{H} 1975$ were gifted by Henan Institute of Medical and Pharmaceutical Sciences, Zhengzhou University.

\section{Reagents}

Antibodies used included BCAT1 (Sangon Biotech, China), phospho-NF- $k$ B p65 (Ser536) (CST, USA), IKKa/ $\beta$ (Wanleibio, China), IKB $a$ (Wanleibio, China), p65 (Wanleibio, China), GAPDH (Sangon Biotech, China), $\beta$-Actin (proteintech, China), E-cadherin (proteintech, China), Vimentin (proteintech, China), Antibody cocktail to aerobic respiration (CST, USA). PDTC, L-Valine, L-Isoleucine, and L-Leucine (MCE, USA).

\section{Immunohistochemistry (IHC) and Evaluation}


The tissue microarrays (TMAs) consisting of 86 pairs of human LUAD and normal lung tissues were purchased from Outdo Biotech (China). This study was conducted with the informed consent obtained from all subjects. All experiments were performed in accordance with relevant guidelines and regulations of Declaration of Helsinki and has been approved by the Research Ethics Committee of Zhengzhou University. Anti-BCAT1 antibody was used for IHC detection of BCAT1 expression according to the manufacturer's suggested protocols. In brief, paraffin sections were dewaxed by heat treatment in EDTA buffer, $\mathrm{pH}=9$. After blocking for $30 \mathrm{~min}$ with $3 \%$ bovine serum albumin (BSA), tissue sections were incubated with anti-BCAT1 antibody diluted 1:400 overnight at $4{ }^{\circ} \mathrm{C}$. Then they were incubated with the secondary antibody (HRP labeled) at room temperature for $50 \mathrm{~min}$. The staining intensity of tissues was scored as 0 (negative), $1(1+), 2(2+), 3(3+)$. For statistical evaluation, tissues were scored as 0 (nonstaining); 1, 1-25\%; 2, 26-50\%; 3, 51-75\%; 4, 76-100\% (positive staining). BCAT1 protein expression was evaluated as negative $(0)$, weak positive $(1,2)$, positive $(3,4)$ according to the staining positive rate score. The total histological score was defined as the product of "staining intensity score" and "staining positive rate score", and 0-5 indicated BCAT1 low expression while 6-12 indicated BCAT1 high expression.

\section{Lentivirus transduction and Stable cell line construction}

BCAT1 overexpression, shRNA and control lentiviral particles were purchased from Genechem and Hanbio Biotechnology (China). The shRNA sequences targeting BCAT1 were as follows: 5GGATCAAGAATGGGTCCCATATTCA-3', The shRNA control sequence was: 5'-

TTCTCCGAACGTGTCACGTAA-3. A549 cells were seeded in 6-well plates and infected with BCAT1 overexpression, shRNA or control lentivirus respectively, followed by incubation overnight with $5 \mu \mathrm{g} / \mathrm{mL}$ polybrene (Sigma-Aldrich, USA). Stable BCAT1 knockdown and overexpression cells were selected with 2 $\mu \mathrm{g} / \mathrm{mL}$ puromycin.

\section{Protein extraction and Western blot}

Cells were lysed in RIPA buffer (Solarbio, China) for $30 \mathrm{~min}$ at $4^{\circ} \mathrm{C}$ and then centrifuged at $12000 \mathrm{rpm}$ for $15 \mathrm{~min}$. The supernatant was collected and total protein was quantified using the BCA kit (Solarbio, China). Total protein was boiled and separated by $10 \%$ SDS-PAGE gel, and then transferred to PVDF membrane (Merck Millipore, USA). Membranes were blocked in 5\% skim milk powder in TBS-T (TBS plus $0.5 \%$ Tween-20) at room temperature for 2 hours, and then blots were incubated with primary antibody at $4^{\circ} \mathrm{C}$ overnight. The membrane was probed with secondary antibody, and signals were detected with ECL Substrate (Beyotime Biotech, China) and Amersham Imager 600 System (General Electric Company, USA). Protein expression was quantified using Image $\mathrm{J}$ software, with GAPDH or $\beta$-Actin as an internal control.

\section{RNA Extraction and quantitative real-time PCR (qRT-PCR)}


Total RNA was extracted from cells with TRIzol Reagent (Invitrogen, USA) according to the manufacturer's instruction and cDNA was synthesized using the PrimeScript RT Reagent Kit (TaKaRa Clontech, China). qRT-PCR was performed using the ChamQ ${ }^{\mathrm{TM}}$ Universal SYBR ${ }^{\circledR}$ qPCR Master Mix (Vazyme Biotech, China) and QuantStudio 5 Real-Time PCR System (Thermo Scientific, USA). The target mRNA expression level was quantified using the $2^{-\triangle \Delta C t}$ method with normalized to the endogenous gene ACTB. The following primer sequences were used for qRT-PCR: BCAT1 forward, 5-TGGAGAATGGTCCTAAGCTG-3', reverse, 5GCACAATTGTCCAGTCGCT C-3; NFKBIB forward, 5'-GCCCTGACCGTACTCCCGAC-3', reverse, 5'-GGCCTCC ACTGCCAAATGAA-3; BCKDK forward, 5'-CTTCTGAAAAGTGCTCGGTAC-3', reverse, 5'-

CTTCTGGAAGGCACGGATATAT-3; BCKDHA forward, 5'-GAGGCAGG TGTGCTGATGTATCG-3', reverse, 5' AGTGGAGAGGAGATAGTGACGAAGTG-3; BCKDHB forward, 5-GGTGGCTCATTTTACTTTCCAG-3; reverse, 5-AGGATCTTT GGCCAATGAGTTA-3; ECHS1 forward, 5'-GTGATATCATCTATGCCGGTGA-3', reverse, 5GTCTCAACAGGACAAATCTTGC-3; GAPDH forward, 5'-CTCAAGGG CATCCTGGGCTA-3', reverse, 5' CGTCAAAGGTGGAGGAGTGG-3; ACTB forward, 5'-GGCATCCACGAAACTACCTT-3', reverse, 5'CTCCTGCTTGCTGATCCACA -3. Primers used for qRT-PCR were synthesized by Shenggong, China.

\section{RNA sequencing (RNA-seq) and Informatics analysis}

RNA-seq was performed with BCAT1 overexpression and control A549 cells (each with three replicates) using BGISEQ-500 sequencing system. In brief, RNA sample was extracted and the quality was assessed, followed by generation of cDNA libraries. Gene expression levels for each sample were quantified using the FPKM method (fragments per kilobase of transcript per million mapped reads) and processed for analysis. DEGs were screened by fold change $\geq 1.5$ and the adjusted $p$ value $\leq 0.01$. Gene Ontology (GO) annotation proteome was performed from the UniProt-GOA database (http://www.ebi.ac.uk/GOA). Kyoto Encyclopedia of Genes and Genomes (KEGG) pathway analysis was based on database (http://www.genome.jp/kegg/).

\section{Cell proliferation assay}

The cell proliferation ability was measured by using the xCELLigence Real-Time Cell Analyzer (RTCA)-MP system (Acea Biosciences, USA), which provided a continuous and quantitative measurement of the cell index to monitor cellular growth status. First, $50 \mu \mathrm{L}$ of culture medium was added in each well of E-Plate 12 (Roche Applied Science, USA) to detect the baseline. Then cells were suspended in $100 \mu \mathrm{L}$ medium and seeded in E-Plate 12 with a density of $5 \times 10^{4}$ cells $/ \mathrm{mL}$. After $30 \mathrm{~min}$, the E-Plate 12 was inserted into RTCA-MP device and incubated at $37^{\circ} \mathrm{C}$ with $5 \% \mathrm{CO}_{2}$. Cell index was measured every 5 min to reflect the cellular proliferation ability.

\section{CCK-8 assay}


Cells in the logarithmic growth phase were seeded in a 96-well plate (3000 cells/well), and the total volume of cell suspension per well was $100 \mu \mathrm{L}$. After incubation for $24 \mathrm{~h}$ and $48 \mathrm{~h}, 10 \mu \mathrm{L}$ CCK-8 reagent was added into each well, and then cells were incubated for $1-4 \mathrm{~h}$ at $37^{\circ} \mathrm{C}$ in the dark. The absorbance (A) value at $450 \mathrm{~nm}$ wavelength was detected with a microplate reader (Thermo, USA).

\section{Colony formation assay}

Cells were seeded into 6-well plates ( 1000 cells/well). Then cells were cultured at $37^{\circ} \mathrm{C}$ with $5 \% \mathrm{CO}_{2}$ for approximately one week. After that, clones were washed with PBS and then fixed with $4 \%$ paraformaldehyde, followed by staining with $1 \%$ crystal violet for $30 \mathrm{~min}$. The colonies were examined and counted with a microscope.

\section{Wound-healing assay}

Cells were seeded with a density of $10^{6}$ cells/well in 6-well plates, when the cell density reached $95 \%$, monolayers were scratched with a $10 \mu \mathrm{L}$ pipette tip to create scratch wounds. The suspended cells were washed away with PBS, and then cultured in RPMI- 1640 medium with $2 \%$ FBS. The distance between scratches was measured at $0 \mathrm{~h}, 12 \mathrm{~h}, 24 \mathrm{~h}$, and $48 \mathrm{~h}$, and the relative residual area (\%) was calculated using the following equation: (current gap distance/original gap distance) $\times 100 \%$.

\section{Cell migration and invasion assays}

For migration assay, $5 \times 10^{4}$ cells were suspended in serum-free medium and seeded in the top chambers, then the lower chambers were filled with $600 \mu \mathrm{L}$ medium containing 10\% FBS. After incubation for $8 \sim 12$ $\mathrm{h}$, the migratory cells were fixed with methanol and stained with a $0.1 \%$ crystal violet solution. Then cells were counted with a microscope (magnification, x200; Olympus BX53, Japan). For invasion assay, the difference was that Matrigel (BD Biosciences, USA) was diluted in serum-free medium (1:6) and added to the upper chamber before cells were seeded.

\section{Xenograft mice model}

Animal study was performed in accordance with the guidelines of the National Act on the Use of Laboratory Animals (P. R. China), approved by the Animal Ethics Committee of Zhengzhou University. This study was reported in accordance with ARRIVE guidelines. BALB/c nude mice (female, aged 4 weeks) were upraised in SPF conditions in Henan Key Laboratory for Pharmacology of liver diseases, with a $12 \mathrm{~h}$ light/dark cycle and free access to food and water. BCAT1 overexpression and control A549 cells $\left(5 \times 10^{6}\right.$ in $100 \mu \mathrm{L}$ PBS) were subcutaneously injected into the flanks of mice, respectively. Five weeks 
after injection, mice were sacrificed by cervical dislocation and the tumors were collected for analysis. Tumor volume was evaluated with the formula: tumor volume $=\left(\right.$ width $^{2} \mathrm{x}$ length $) / 2$.

\section{Cellular oxygen consumption rate (OCR) detection}

Briefly, cells $\left(8 \times 10^{4}\right)$ were seeded into a 96-well plate and then cultured at $37^{\circ} \mathrm{C}$ with $5 \% \mathrm{CO}_{2}$ for overnight. Next, the BBoxiProbeTM R01 (BestBio, China) and oxygen blocking fluid were added into the 96-well plate chambers in sequence at $37^{\circ} \mathrm{C}$, and the fluorescence intensity at the excitation wavelength of $468 \mathrm{~nm}$ was detected by the CLARIOstar Plus (BMG, Germany).

\section{Statistical analysis}

All statistical analyses were performed using SPSS 21.0 and visualized with GraphPad Prism 6.0. Data are shown as mean \pm standard error of measurement (SEM). A $p$ value $\leq 0.05$ was considered as statistically significant.

\section{Declarations}

\section{Acknowledgments}

We appreciate the continuous support of Henan Key Laboratory for Pharmacology of Liver Diseases where most of the experiments were performed.

\section{Author contributions}

Q.Z., J.Z., and J.Z. designed the study. J.L., M.Y., and Z.Z. performed the experiments and analyzed data. F.X., Y.L., L.D., and B.Z. collected materials and helped in manuscript preparing. Q.Z., M.Y. and J.L. wrote the manuscript. All authors contributed to the article and approved the submitted version.

\section{Conflict of interest}

The authors declare that the research was conducted in the absence of any commercial or financial relationships that could be construed as a potential conflict of interest.

\section{Additional information}

The RNA Sequencing data was submitted to BioSample database of NCBI (BioProject ID: PRJNA717911). 


\section{Ethical statement}

This study was conducted with the informed consent obtained from all subjects. All experiments were performed in accordance with relevant guidelines and regulations of Declaration of Helsinki and has been approved by the Research Ethics Committee of Zhengzhou University.

\section{References}

1. Zhao, X. et al. LncRNA HOXA11-AS drives cisplatin resistance of human LUAD cells via modulating miR-454-3p/Stat3. Cancer Sci 109, 3068-3079, https://doi.org/10.1111/cas.13764 (2018).

2. VandenBussche, C. J., Illei, P. B., Lin, M.-T., Ettinger, D. S. \& Maleki, Z. Molecular alterations in nonsmall cell lung carcinomas of the young. Human Pathology 45, 2379-2387, https://doi.org/10.1016/j.humpath.2014.08.005 (2014).

3. Wu, C. et al. Correlation between serum IL-1 beta and miR-144-3p as well as their prognostic values in LUAD and LUSC patients. Oncotarget 7, 85876-85887, https://doi.org/10.18632/oncotarget.13042 (2016).

4. Cui, Y. et al. Development and Validation of a Novel Signature to Predict Overall Survival in "Driver Gene-negative" Lung Adenocarcinoma (LUAD): Results of a Multicenter Study. Clin Cancer Res 25, 1546-1556, https://doi.org/10.1158/1078-0432.CCR-18-2545 (2019).

5. Zhao, X. et al. Overexpression of KIF20A confers malignant phenotype of lung adenocarcinoma by promoting cell proliferation and inhibiting apoptosis. Cancer Med 7, 4678-4689, https://doi.org/10.1002/cam4.1710 (2018).

6. Zhang, J., Sun, G. \& Mei, X. Elevated FAM83A expression predicts poorer clincal outcome in lung adenocarcinoma. Cancer Biomark 26, 367-373, https://doi.org/10.3233/CBM-190520 (2019).

7. Zhang, J. et al. Overexpression of FAM83H-AS1 indicates poor patient survival and knockdown impairs cell proliferation and invasion via MET/EGFR signaling in lung cancer. Sci Rep 7, 42819, https://doi.org/10.1038/srep42819 (2017).

8. Wang, X., He, M., Li, J., Wang, H. \& Huang, J. KLF15 suppresses cell growth and predicts prognosis in lung adenocarcinoma. Biomed Pharmacother 106, 672-677,

https://doi.org/10.1016/j.biopha.2018.07.006 (2018).

9. Luo, J. et al. PITX2 enhances progression of lung adenocarcinoma by transcriptionally regulating WNT3A and activating Wnt/beta-catenin signaling pathway. Cancer Cell Int 19, 96, https://doi.org/10.1186/s12935-019-0800-7 (2019).

10. Dimitrakopoulos, F. I. et al. NSCLC and the alternative pathway of NF-kappaB: uncovering an unknown relation. Virchows Arch 460, 515-523, https://doi.org/10.1007/s00428-012-1230-2 (2012).

11. Wu, C. et al. IL-1beta-Mediated Up-Regulation of WT1D via miR-144-3p and Their Synergistic Effect with NF-kappaB/COX-2/HIF-1 alpha Pathway on Cell Proliferation in LUAD. Cell Physiol Biochem 48, 2493-2502, https://doi.org/10.1159/000492687 (2018). 
12. Ananieva, E. A. \& Wilkinson, A. C. Branched-chain amino acid metabolism in cancer. Curr Opin Clin Nutr Metab Care 21, 64-70, https://doi.org/10.1097/MC0.0000000000000430 (2018).

13. Mayers, J. R. \& Vander Heiden, M. G. Nature and Nurture: What Determines Tumor Metabolic Phenotypes? Cancer Res 77, 3131-3134, https://doi.org/10.1158/0008-5472.CAN-17-0165 (2017).

14. Xie, F. et al. Reciprocal control of IncRNA-BCAT1 and beta-catenin pathway reveals IncRNA-BCAT1 long non-coding RNA acts as a tumor suppressor in colorectal cancer. Oncotarget 8, 23628-23637, https://doi.org/10.18632/oncotarget.15466 (2017).

15. Xu, Y. et al. Overexpression of BCAT1 is a prognostic marker in gastric cancer. Hum Pathol 75, 41-46, https://doi.org/10.1016/j.humpath.2018.02.003 (2018).

16. Zhang, L. \& Han, J. Branched-chain amino acid transaminase 1 (BCAT1) promotes the growth of breast cancer cells through improving mTOR-mediated mitochondrial biogenesis and function. Biochem Biophys Res Commun 486, 224-231, https://doi.org/10.1016/j.bbrc.2017.02.101 (2017).

17. Zhu, W., Shao, Y. \& Peng, Y. MicroRNA-218 inhibits tumor growth and increases chemosensitivity to CDDP treatment by targeting BCAT1 in prostate cancer. Mol Carcinog 56, 1570-1577, https://doi.org/10.1002/mc.22612 (2017).

18. Oktyabri, D., Ishimura, A., Tange, S., Terashima, M. \& Suzuki, T. DOT1L histone methyltransferase regulates the expression of BCAT1 and is involved in sphere formation and cell migration of breast cancer cell lines. Biochimie 123, 20-31, https://doi.org/10.1016/j.biochi.2016.01.005 (2016).

19. Wang, Z.-Q. et al. BCAT1 expression associates with ovarian cancer progression: possible implications in altered disease metabolism. Oncotarget 6, 31522-31543, https://doi.org/10.18632/oncotarget.5159 (2015).

20. Zheng, Y. H. et al. BCAT1, a key prognostic predictor of hepatocellular carcinoma, promotes cell proliferation and induces chemoresistance to cisplatin. Liver Int 36, 1836-1847, https://doi.org/10.1111/liv.13178 (2016).

21. Fu, S. et al. Glutamine Synthetase Promotes Radiation Resistance via Facilitating Nucleotide Metabolism and Subsequent DNA Damage Repair. Cell Rep 28, 1136-1143 e1134, https://doi.org/10.1016/j.celrep.2019.07.002 (2019).

22. Lin, X., Tan, S., Fu, L. \& Dong, Q. BCAT1 Overexpression Promotes Proliferation, Invasion, and Wnt Signaling in Non-Small Cell Lung Cancers. Onco Targets Ther 13, 3583-3594, https://doi.org/10.2147/0TT.S237306 (2020).

23. Wang, P. et al. BCAT1 promotes proliferation of endometrial cancer cells through reprogrammed BCAA metabolism. International Journal of Clinical and Experimental Pathology 11, 5536-+, (2018).

24. Tonjes, M. et al. BCAT1 promotes cell proliferation through amino acid catabolism in gliomas carrying wild-type IDH1. Nat Med 19, 901-908, https://doi.org/10.1038/nm.3217 (2013).

25. Papathanassiu, A. E. et al. BCAT1 controls metabolic reprogramming in activated human macrophages and is associated with inflammatory diseases. Nat Commun 8, 16040, https://doi.org/10.1038/ncomms16040 (2017). 
26. Fukami, K., Yamagishi, S.-i. \& Okuda, S. Role of AGEs-RAGE System in Cardiovascular Disease. Current Pharmaceutical Design 20, 2395-2402, https://doi.org/10.2174/13816128113199990475 (2014).

27. Rai, A. K. et al. Fructose-induced AGEs-RAGE signaling in skeletal muscle contributes to impairment of glucose homeostasis. J Nutr Biochem 71, 35-44, https://doi.org/10.1016/j.jnutbio.2019.05.016 (2019).

28. Zhou, W. et al. Over-expression of BCAT1, a c-Myc target gene, induces cell proliferation, migration and invasion in nasopharyngeal carcinoma. Molecular Cancer 12, https://doi.org/10.1186/14764598-12-53 (2013).

29. Shamekhi, S. et al. Apoptotic Effect of Saccharomyces cerevisiae on Human Colon Cancer SW480 Cells by Regulation of Akt/NF-kB Signaling Pathway. Probiotics Antimicrob Proteins 12, 311-319, https://doi.org/10.1007/s12602-019-09528-7 (2020).

30. Zhang, Z., Ma, J., Li, N., Sun, N. \& Wang, C. Expression of nuclear factor-kappaB and its clinical significance in nonsmall-cell lung cancer. Ann Thorac Surg 82, 243-248, https://doi.org/10.1016/j.athoracsur.2006.01.049 (2006).

31. Wu, D. et al. NF-kappaB Expression and Outcomes in Solid Tumors: A Systematic Review and MetaAnalysis. Medicine (Baltimore) 94, e1687, https://doi.org/10.1097/MD.0000000000001687 (2015).

32. Cai, H., Yan, L., Liu, N., Xu, M. \& Cai, H. IFI16 promotes cervical cancer progression by upregulating PD-L1 in immunomicroenvironment through STING-TBK1-NF-kB pathway. Biomedicine \& Pharmacotherapy 123, https://doi.org/10.1016/j.biopha.2019.109790 (2020).

33. Zhang, T. et al. MicroRNA-378 promotes hepatic inflammation and fibrosis via modulation of the NFkappaB-TNFalpha pathway. J Hepatol 70, 87-96, https://doi.org/10.1016/j.jhep.2018.08.026 (2019).

34. Senga, S., Kobayashi, N., Kawaguchi, K., Ando, A. \& Fujii, H. Fatty acid-binding protein 5 (FABP5) promotes lipolysis of lipid droplets, de novo fatty acid (FA) synthesis and activation of nuclear factor-kappa B (NF-kappaB) signaling in cancer cells. Biochim Biophys Acta Mol Cell Biol Lipids 1863, 1057-1067, https://doi.org/10.1016/j.bbalip.2018.06.010 (2018).

35. Chen, L. et al. Design and optimize N-substituted EF24 as effective and low toxicity NF-kappaB inhibitor for lung cancer therapy via apoptosis-to-pyroptosis switch. Chem Biol Drug Des 94, 13681377, https://doi.org/10.1111/cbdd.13514 (2019).

36. Gallagher, S. J. et al. Control of NF-kB activity in human melanoma by bromodomain and extraterminal protein inhibitor I-BET151. Pigment Cell Melanoma Res 27, 1126-1137, https://doi.org/10.1111/pcmr.12282 (2014).

37. Tyagi, M. \& Patro, B. S. Salinomycin reduces growth, proliferation and metastasis of cisplatin resistant breast cancer cells via NF-kB deregulation. Toxicol In Vitro 60, 125-133, https://doi.org/10.1016/j.tiv.2019.05.004 (2019).

38. Wu, K. J. et al. Small Molecule Pin1 Inhibitor Blocking NF-kappaB Signaling in Prostate Cancer Cells. Chem Asian J 13, 275-279, https://doi.org/10.1002/asia.201701216 (2018). 
Figures

A

$10 \mathrm{X}$

$100 \mathrm{X}$
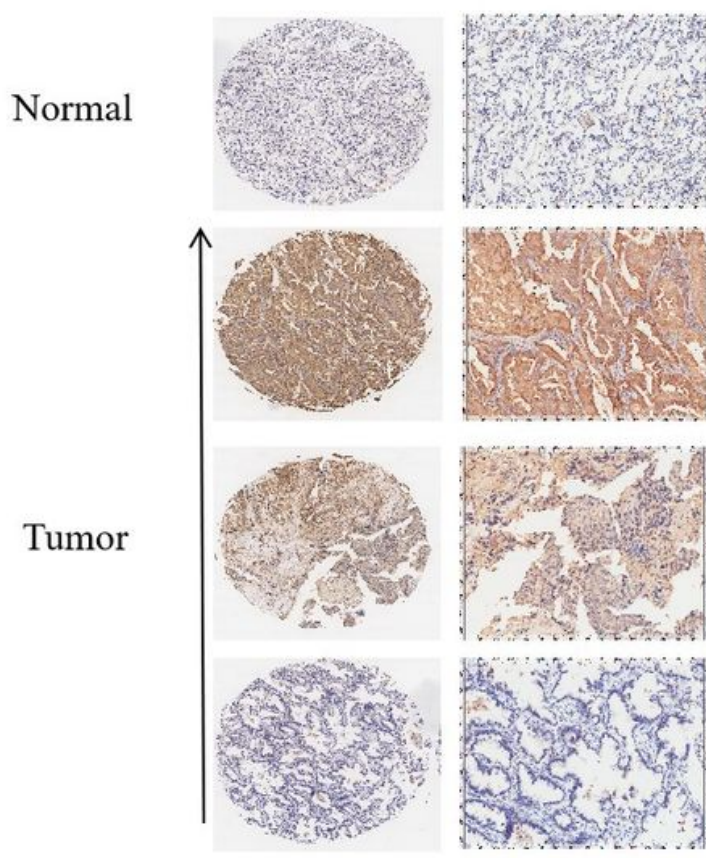

Negative
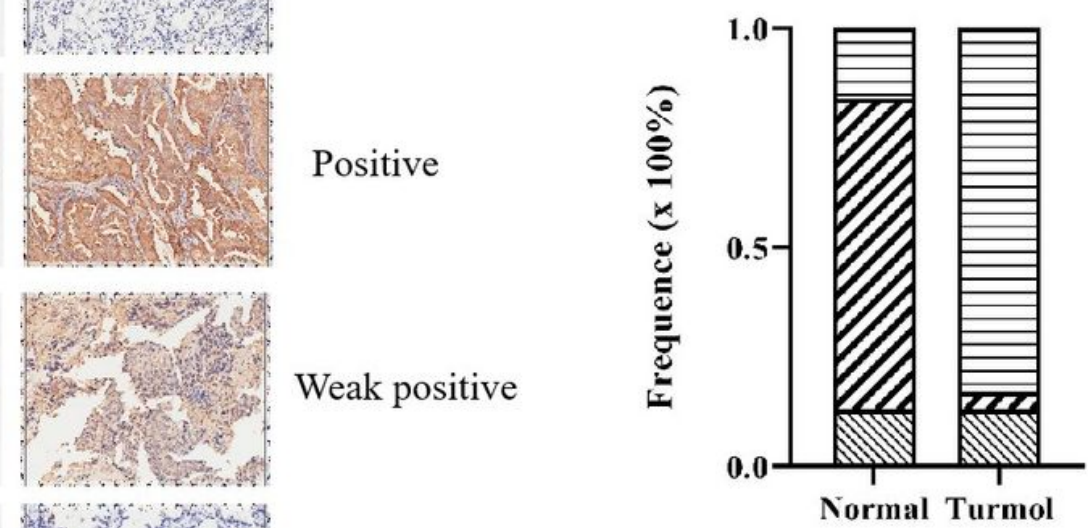

曰 Positive

a Negative

Weak positive

Weak positive

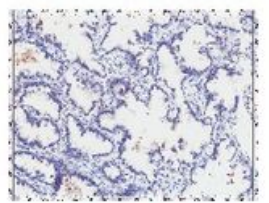

Negative

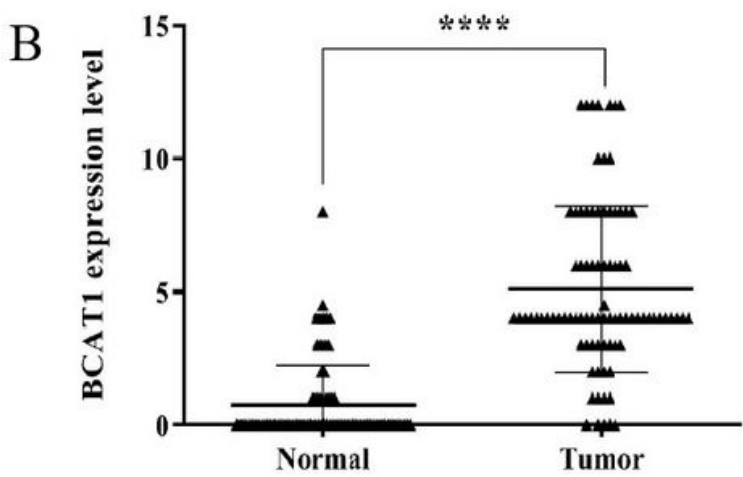

C

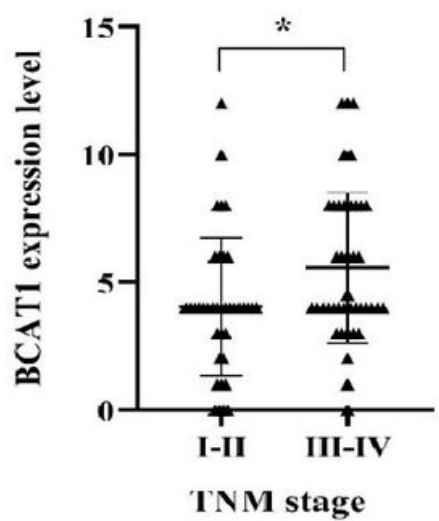

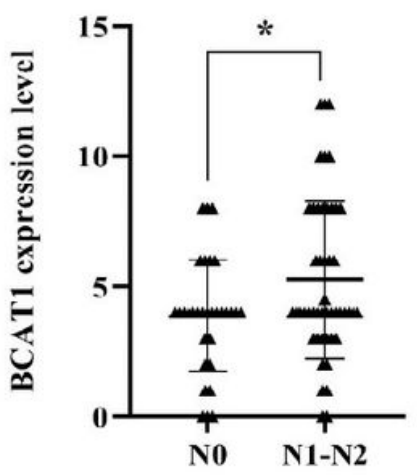

Lymph node metastasis

Figure 1

BCAT1 expression is increased in LUAD tumor tissues. (A) IHC staining of BCAT1 in LUAD tumor and normal lung tissues. Histogram showed the frequency of positive, weak positive and negative staining. (B) Expression levels of BCAT1 in LUAD tumor and normal lung tissues. (C) TNM stage and local lymph node metastasis frequency of LUAD patients with different BCAT1 expression levels. 
A
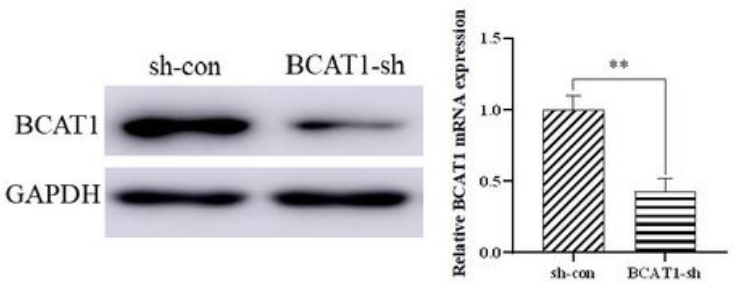

$\mathrm{C}$
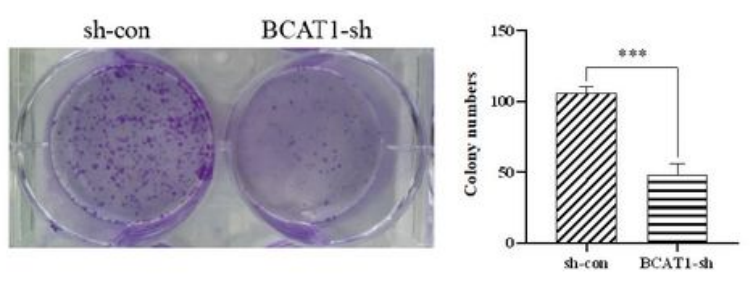

E

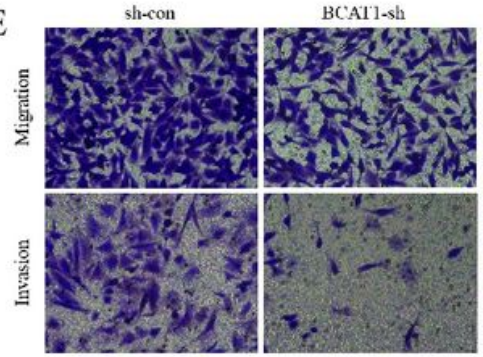

B

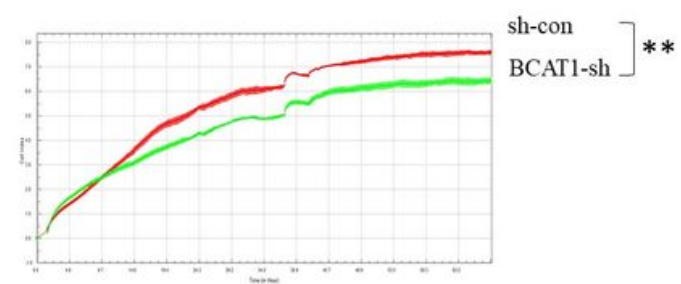

D
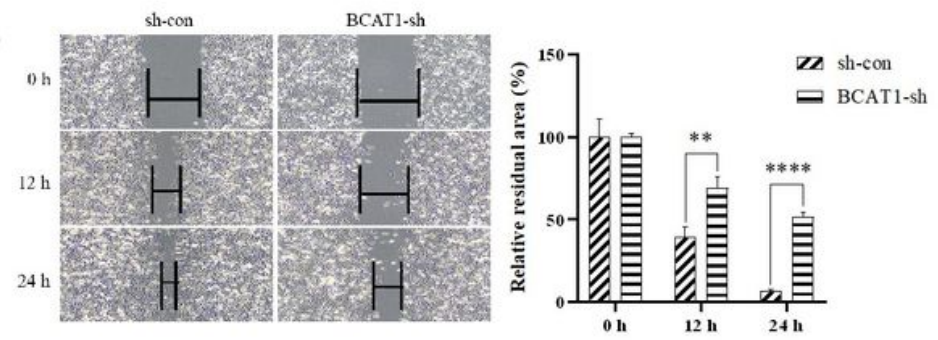

$\mathrm{F}$

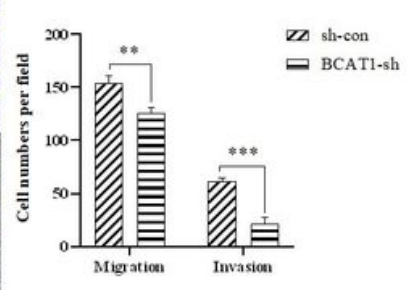

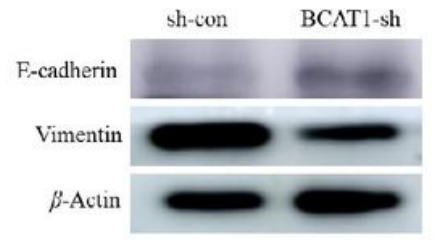

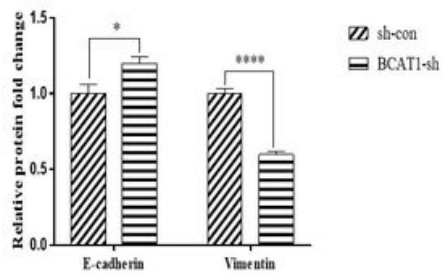

Figure 2

BCAT1 knockdown inhibits cell proliferation, colony formation, migration and invasion. (A) Western blot and qRT-PCR analysis of BCAT1 in A549 cells transfected with sh-con and shRNAs. (B) Cell proliferation rate detection by using the real-time cell electronic sensing system. (C-E) Colony formation, wound healing, migration and invasion assays of A549 cells transfected with sh-con and shRNAs. (F) Western blot analysis of E-cadherin and Vimentin in BCAT1 knockdown and control A549 cells. 
A
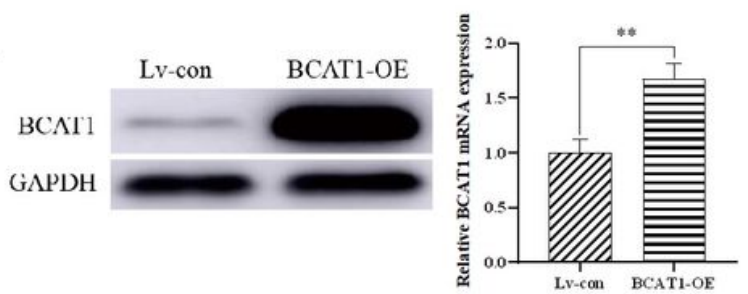

$\mathrm{C}$
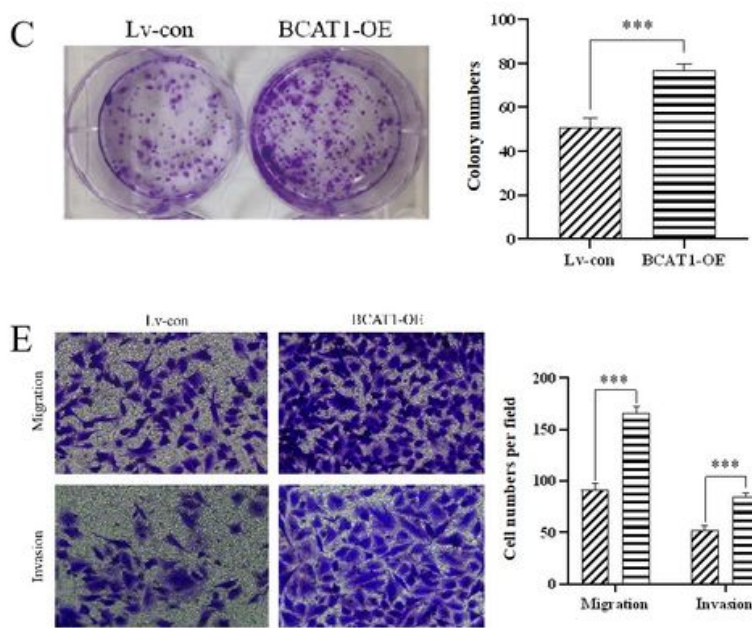

D

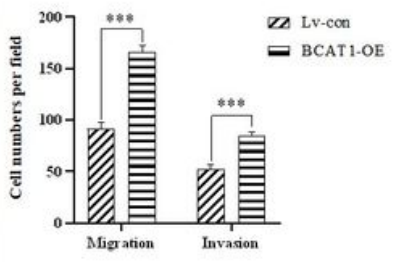

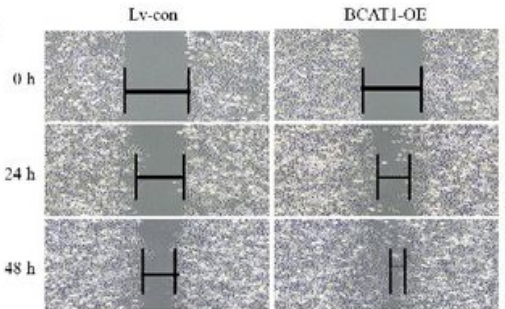

F

B

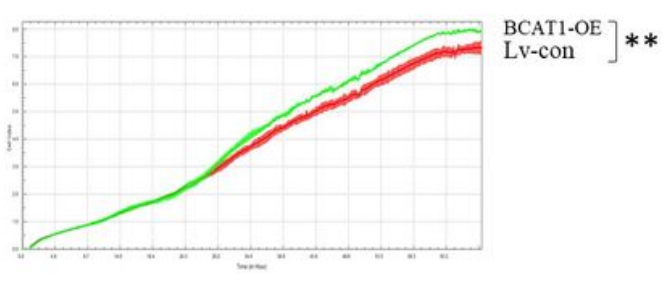

BCATI-OE
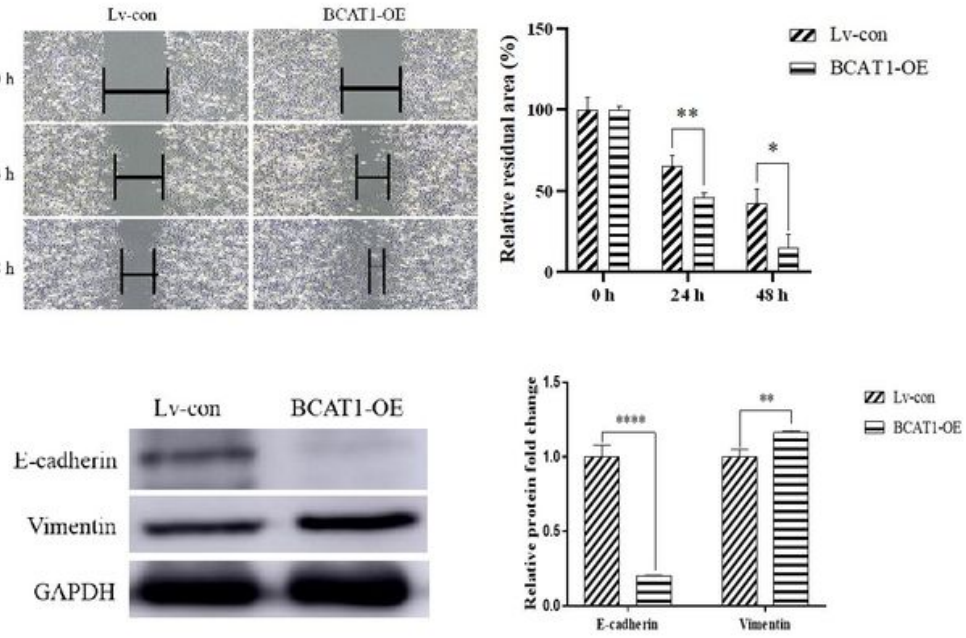

Figure 3

BCAT1 overexpression promotes cell proliferation, colony formation, migration and invasion. (A) Western blot and qRT-PCR analysis of BCAT1 in Lv-con and BCAT1 overexpression A549 cells. (B) Cell proliferation rate detection by using the real-time cell electronic sensing system. (C-E) Colony formation, wound healing, migration and invasion assays of Lv-con and BCAT1 overexpression A549 cells. (F) Western blot analysis of E-cadherin and Vimentin in BCAT1 overexpression and control A549 cells. 
A

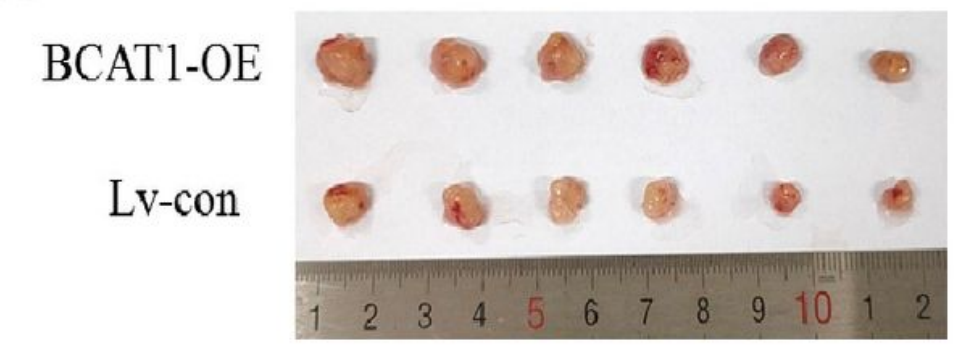

$\mathrm{D}$

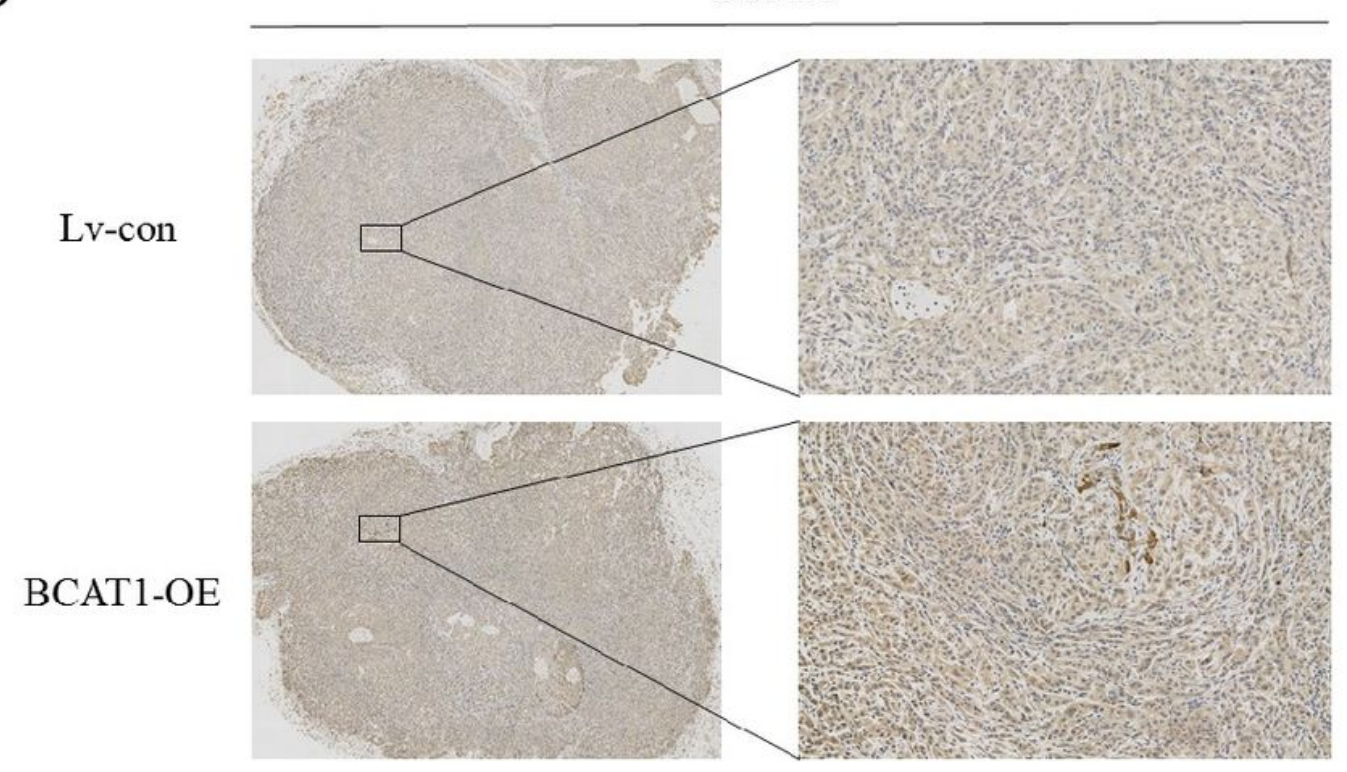

B

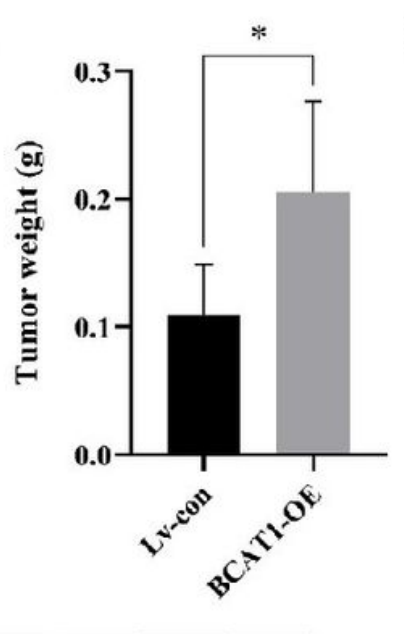

BCAT1
$\mathrm{C}$

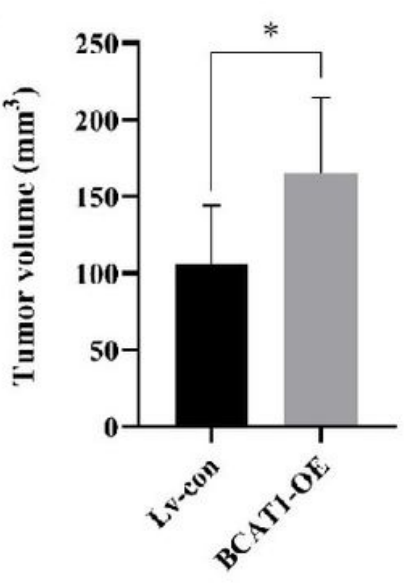

Figure 4

BCAT1 overexpression promotes LUAD tumor formation and growth in vivo. (A) Tumors were established in xenograft mice model after injection with BCAT1 overexpression and control LAUD cells, respectively.

(B) and (C) Tumor weight and volume in xenograft mice model. (D) IHC staining of BCAT1 in LUAD tumors from xenograft mice model. 
A

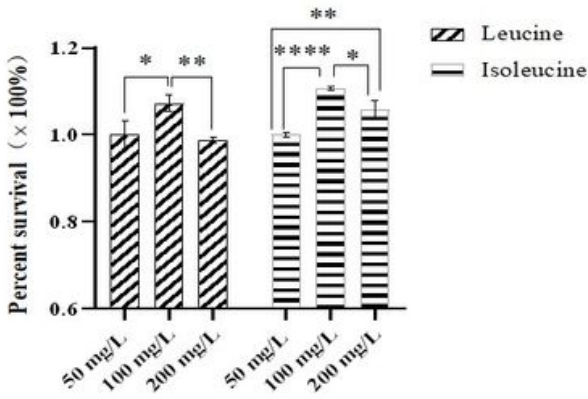

$\mathrm{C}$

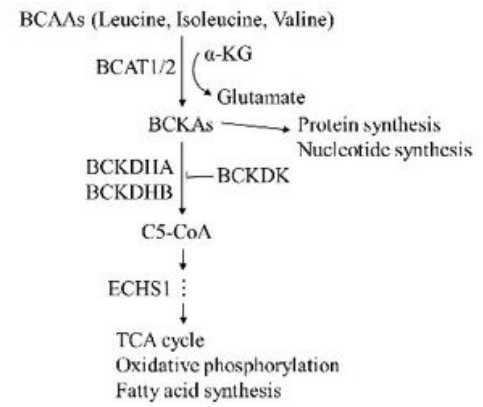

E

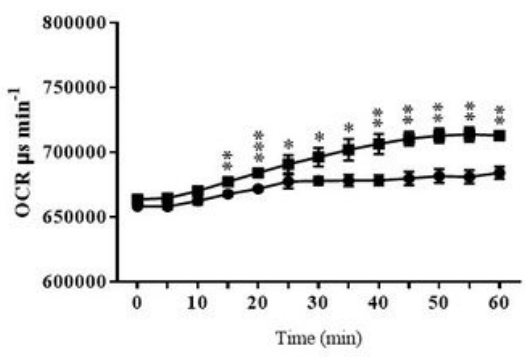

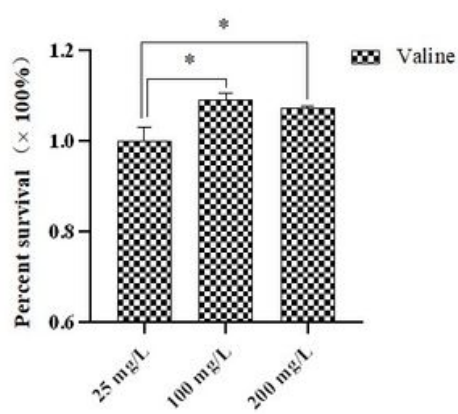

$\mathrm{B}$

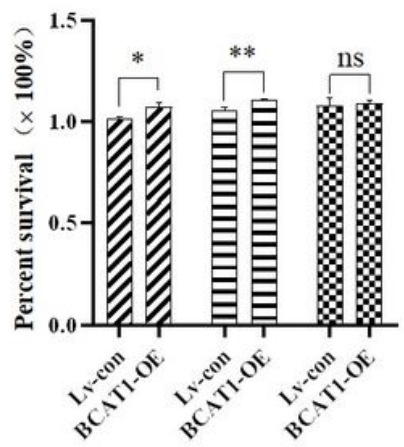

$\mathrm{D}$

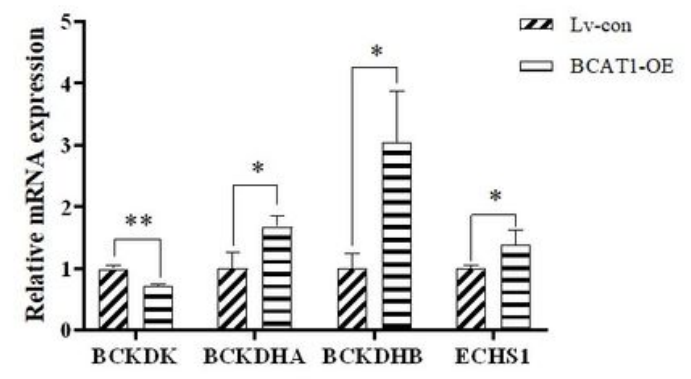

$\rightarrow$ Lv-con

- BCATLOE

$\mathrm{F}$

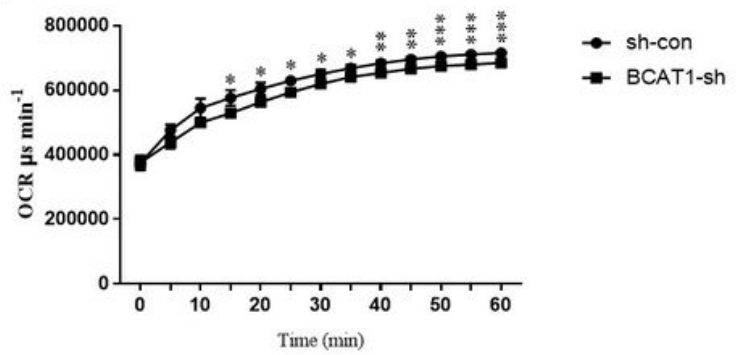

Figure 5

BCAT1 overexpression enhanced BCAAs metabolism and oxygen consumption of LUAD cells. (A) Cell proliferation ability detection of BCAT1 overexpression cells with different kinds and concentrations of BCAAs. (B) Cell proliferation ability detection of BCAT1 overexpression and control cells with different kinds of BCAAs (100 mg/L). (C) Diagram of BCAAs metabolic pathway. (D) qRT-PCR analysis of BCKDK, BCKDHA, BCKDHB, and ECHS1 in BCAT1 overexpression and control A549 cells. (E) and (F) OCR measurement of BCAT1 overexpression, knockdown, and control A549 cells. 
A

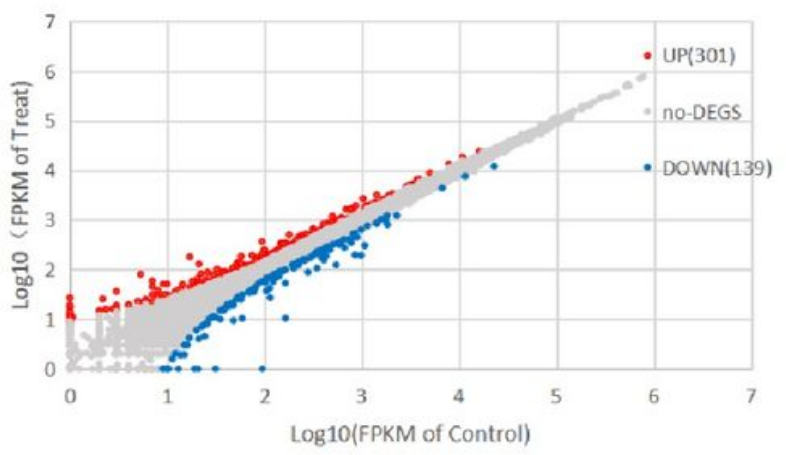

C

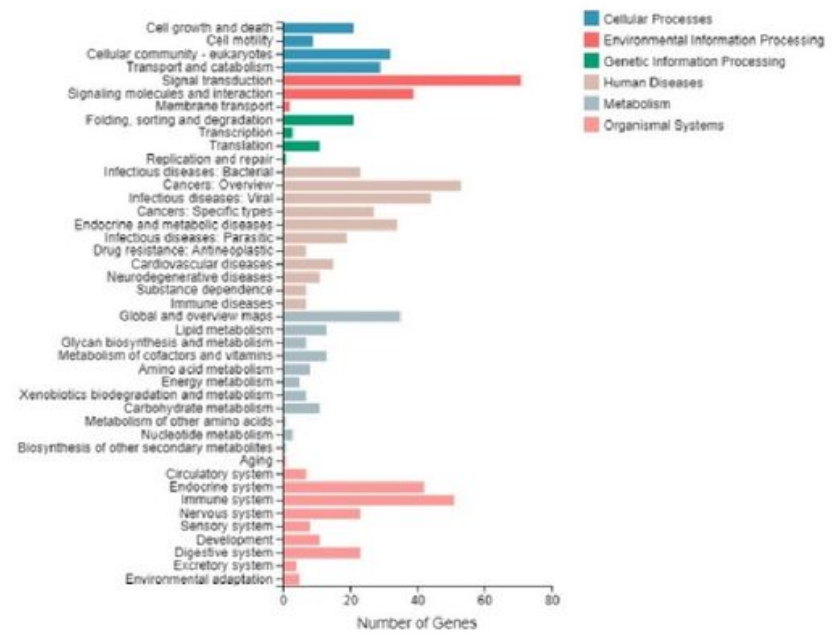

B

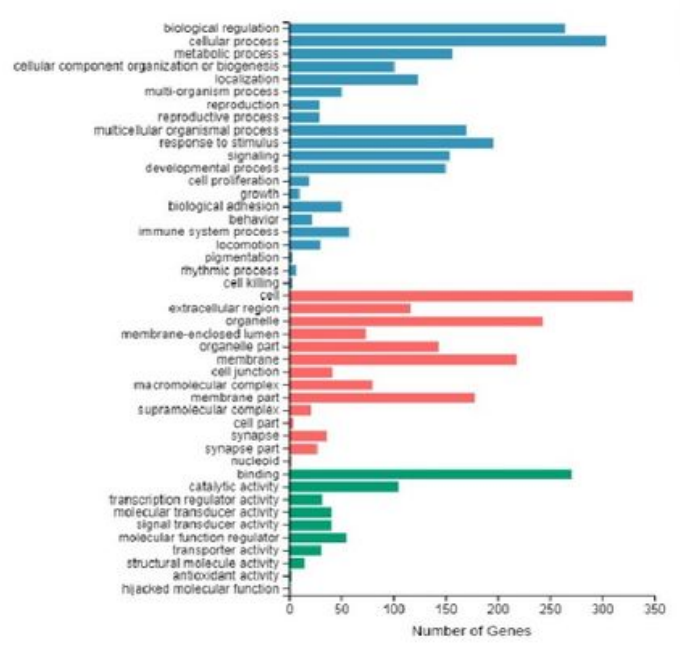

$\mathrm{D}$

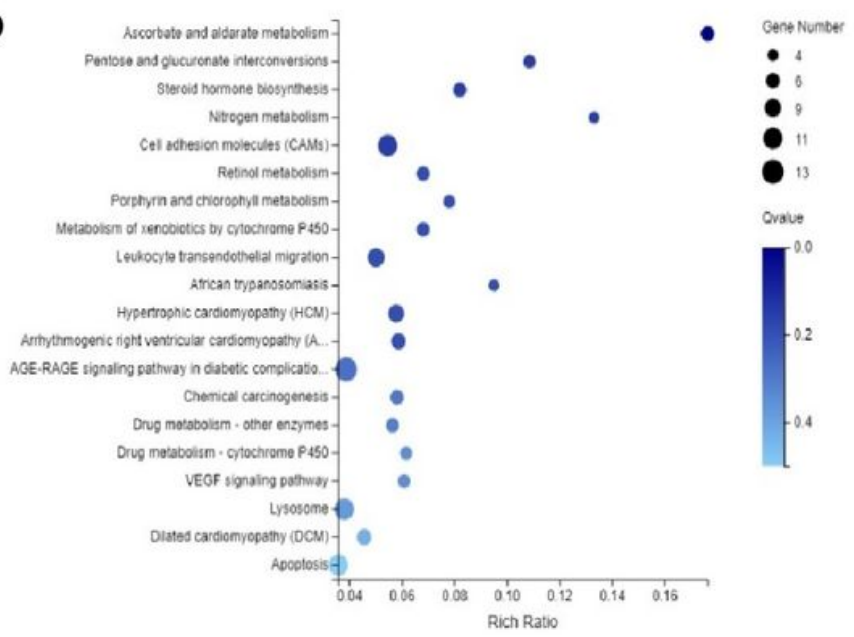

Figure 6

Transcriptome analysis of DEGs induced by BCAT1 overexpression in A549 cells. (A) Scatter plot of global gene expression pattern in Lv-con and BCAT1 overexpression A549 cells. (B) GO functional classification analysis including biological process, cellular component, and molecular function of DEGs. (C) KEGG pathway classification analysis of DEGs based on KEGG database. (D) KEGG functional enrichment analysis of DEGs. 
A

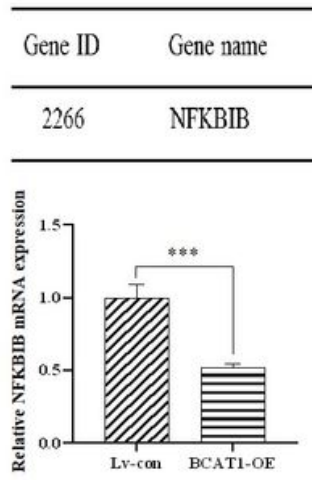

$\mathrm{C}$

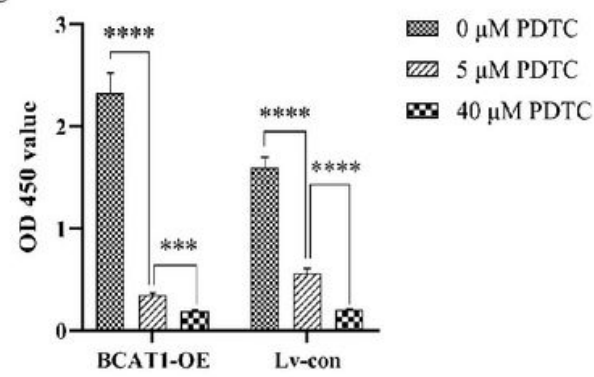

B

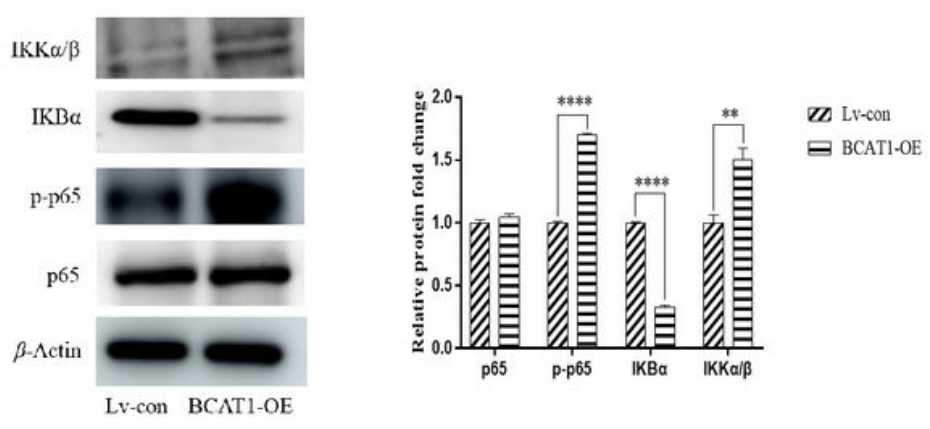

$\mathrm{D}$

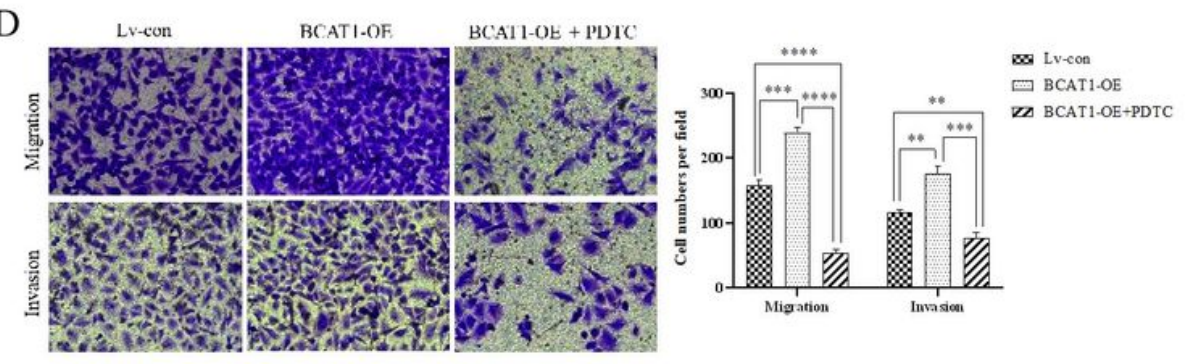

Figure 7

BCAT1 overexpression activates NF-KB pathway. (A) The fold change of NFKBIB mRNA level from RNA sequencing result, and qRT-PCR analysis of NFKBIB in BCAT1 overexpression, knockdown and control A549 cells. (B) Western blot analysis of p65, p-p65, IKBa, and IKKa/ $\beta$ in BCAT1 overexpression and control A549 cells. (C-D) Cell proliferation, migration, and invasion abilities detection of LUAD cells after PDTC treatment. 


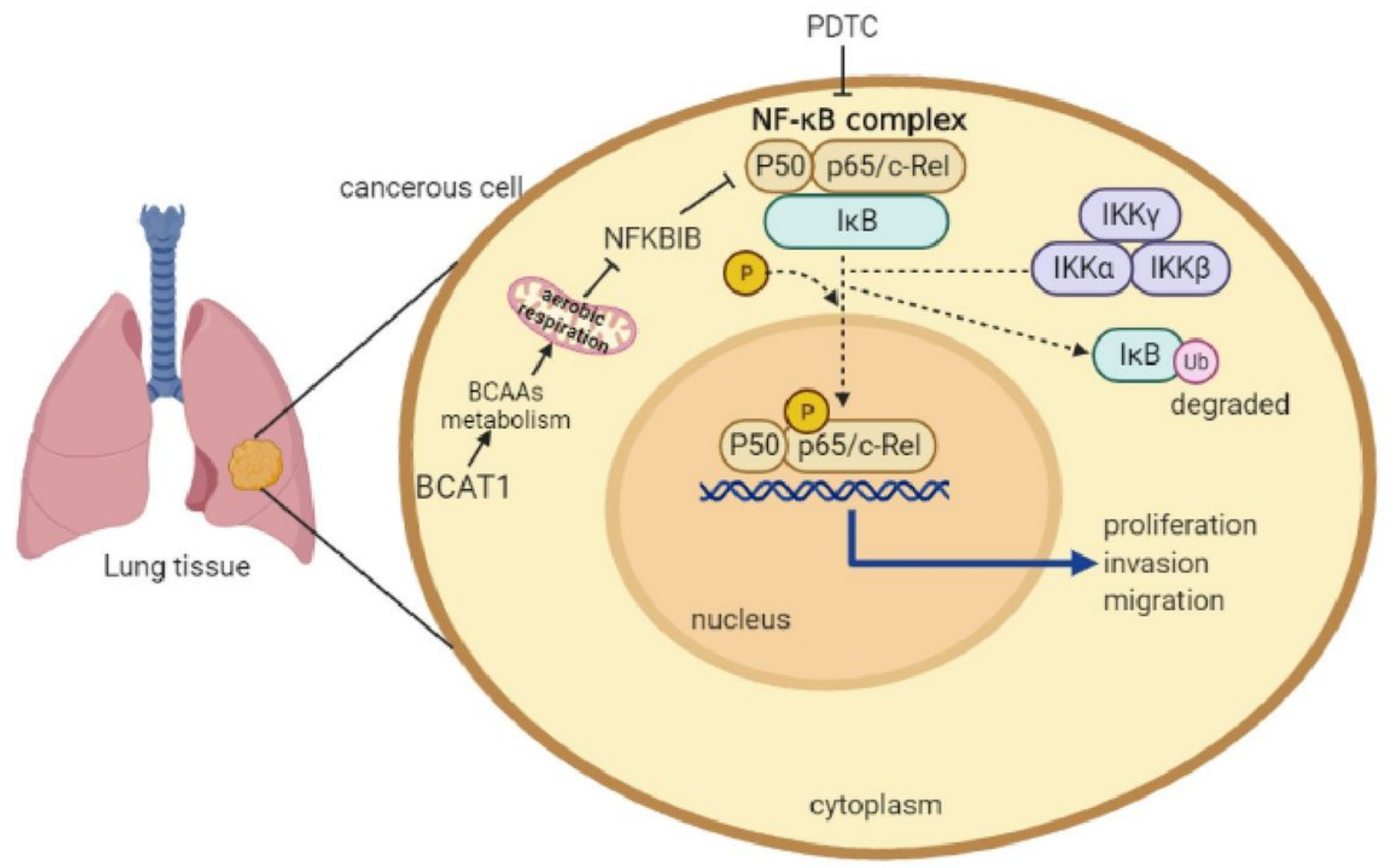

Figure 8

Schematic model of BCAT1 overexpression promotes LUAD development through activating NF-KB pathway, which could be prevented by PDTC treatment.

\section{Supplementary Files}

This is a list of supplementary files associated with this preprint. Click to download.

- Supplementarylnformation.pdf 